\title{
Age and gender specific cut-off points for body fat parameters among adults in Qatar
}

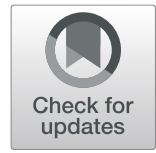

H. Bawadi ${ }^{*}$ (D, S. Hassan ${ }^{1}$, A. Shanbeh Zadeh ${ }^{1}$ H. Sarv ${ }^{1}$, A. Kerkadi ${ }^{1}$, Josep A. Tur ${ }^{2}$ and Z. Shi ${ }^{1}$

\begin{abstract}
Background: Excessive body fat is the leading cause of many metabolic disorders. Therefore, assessing levels of body fat associated with risk of disease in specific populations is crucial. The present study aimed to identify optimal cut-off values of body fat composition including total body fat, body fat percentage, visceral fat, and trunk fat, in order to predict metabolic risk in the Qatari population.

Methods: This cross-sectional study was based on Qatar Biobank data of 2407 Qatari adults (1269 male and 1138 female) aged 21-70 years old. Individuals' height, weight and body fat percentage were obtained. Blood test data including lipid profile, blood glucose and $\mathrm{HbA1c}$ data were also obtained. The area under the curve was calculated using ROC analysis to obtain the body fat percentage associated with risk of disease.

Results: The cut-off points for total fat for those aged $<40$ were $34.0 \mathrm{~kg}$, and for those aged $\geq 40$ were $30.7 \mathrm{~kg}$ and $35.6 \mathrm{~kg}$ in men and women, respectively. The cut-off for body fat percent for those aged $<40$ were 35.1 and $45.1 \%$, and for those aged $\geq 40$ were 34.8 and $46.3 \%$ in men and women, respectively. The cut-off points for trunk fat percent for those aged $<40$ were 19.5 and $22.4 \%$, and for those aged $\geq 40$ were 21.6 and $23.4 \%$ in men and women, respectively. The cut-off points for visceral fat percent for those aged $<40$ were 1.4 and $1.0 \%$, and for those aged $\geq 40$ were 1.9 and $1.4 \%$ in men and women, respectively.

Conclusion: This study established Qatari adult-specific cut-off values of body fat for different age and gender groups.

Keywords: Body fat, DXA, Cut-off, Qatar Biobank
\end{abstract}

\section{Background}

The global prevalence of obesity nearly doubled between 1980 and 2008. The World Health Organization reported that $35 \%$ of adults worldwide aged over 20 years were overweight (34\% men and 35\% women) including 10\% men and $14 \%$ women being considered as obese [1]. The prevalence of overweigh and obesity among Qatari population has reached an alarming rate of 70 and $41 \%$ respectively [2]. It is well known that the accumulation of excessive body fat may contribute to the development of cardiovascular risk factors, such as hypertension, insulin resistance, diabetes mellitus, and dyslipidaemia which

\footnotetext{
* Correspondence: hbawadi@qu.edu.qa

'Department of Human Nutrition, College of Health Sciences, QU Health, Qatar University, Doha 2713, Qatar

Full list of author information is available at the end of the article
}

contribute to cardiovascular diseases (CVD), such as coronary heart disease and stroke [3-5].

Accumulation of adipose tissue among obese individuals is an independent underlying cause of several chronic lifethreatening diseases. It is therefore essential to assess body fat $(\mathrm{BF})$ percentage.

Assessing $\mathrm{BF}$ and its distribution would be useful in screening for obesity and its metabolic risk factors [6]. It is well known that levels of body fat associated with metabolic risk vary between ethnic populations, age groups and genders [7]. For instance, results from the Chinese population reported that $\mathrm{BF} \%$ was higher among people with low body mass index (BMI) compared to the European population [8]. Ethnic differences in levels of body fat and metabolic risks emphasise the need to establish population-specific cut-off values for 
body fat. Data investigating body-fat cut-off points associated with metabolic risk in Arabic countries are limited, with no data available in Qatar. Therefore, the overall aim of this study was to establish age and gender specific cut-off points for body fatness indicators, specific for the Qatari population.

\section{Methods}

\section{Participants}

The study sample was based on a subsample of the Qatar Biobank survey. Exclusion criteria of the study were nonQataris, those aged below 21 or above 75 years old, pregnant women, and athletes. A random sample of 2802 was obtained from the Biobank survey data. Participants with incomplete measurements were excluded $(n=218)$. Participants with missing data about unpleasable body fat measurements or metabolic risk indicators were also excluded $(n=177)$. A net sample of 2407 Qatari participants (1269 males and 1138 females) was included in the analysis. Biobank recruitment and data collection protocols were approved by the Hamad Medical Corporation Ethics Committee. Institutional Review Board approval for this study was obtained from Qatar Biobank.

\section{Demographic and health data}

Sociodemographic data were collected as part of a selfcompleted questionnaire. Data about educational level, age, gender, occupation and physical activity were collected. A trained nurse was available to assist participants completing the questionnaire upon request.

Data regarding health, family history and use of medication were collected by face-to-face interview, carried out by a trained nurse. Information about previous and current diseases experienced by the participants or their family members were collected. The interview also included data about the use of prescribed and over-the-counter medication.

Anthropometric measurements including weight, height, and waist and hip circumferences were recorded with participants wearing light clothes [9]. Measurements were taken by a trained registered nurse. For hip and waist circumference measurements, participants were asked to remove outer garments and the circumferences of hip and waist were measured by tape and recorded in centimetres.

\section{Cardio-metabolic data}

Blood samples (approximately $60 \mathrm{ml}$ ) were collected by a registered nurse to measure participants' total cholesterol levels $(\mathrm{CHOL})$, high-density lipoprotein cholesterol (HDL-C), low-density lipoprotein triglycerides (TG), glycosylated glucose (HbA1c\%), and fasting blood glucose. Blood pressure was measured using the Omron 705 automated device. Duplicate measurements were recorded for diastolic and systolic blood pressure measurements, with additional measurements taken if there was a 5 $\mathrm{mmHg}$ or more difference in the duplicate readings.

Metabolic syndrome (MS) was defined using the harmonised international definition [10]. In detail, high waist circumference (WC) was defined as $\mathrm{WC} \geq 102 \mathrm{~cm}$ in men and $\geq 88 \mathrm{~cm}$ in women; low $\mathrm{HDL}$ as $\mathrm{HDL}<1.04$ $\mathrm{mmol} \cdot \mathrm{L}^{-1}$ in men and $<1.29 \mathrm{mmol} \cdot \mathrm{L}^{-1}$ in women; high triglycerides as triglycerides $\geq 1.7 \mathrm{mmol} \cdot \mathrm{L}^{-1}$ or treatment with triglyceride-lowering drugs; high blood pressure as systolic blood pressure $\geq 130 \mathrm{mmHg}$ or diastolic blood pressure $\leq 85 \mathrm{mmHg}$ or treatment with pressurelowering drugs; and high glucose as glucose $\geq 5.6$ $\mathrm{mmol} \cdot \mathrm{L}^{-1}$ or treatment with glucose-lowering drugs, and MS as 3 or more of the above conditions.

\section{Body fat data}

Body composition was measured by full body dual energy X-ray absorptiometry (iDXA, General Electric) scan. Participants were asked to wear a gown and remove jewellery before undergoing the test, so any interference with the X-rays could be avoided. Moreover, participants were asked to lie flat on the scanning table and to stay still so the X-ray image could be taken through the scanning arm without getting blurred.

\section{Statistical analysis}

To calculate the sensitivity, specificity, and area under the curve (AUC) for different adiposity measures in predicting MS, a user-written syntax cutpt in Stata was used. We used the Liu method to maximize the product of the sensitivity and specificity in the selection of the optimal cut point for predicting metabolic syndrome [11]. We calculated the above indicators in men and women. We also stratified the analyses by age groups. All analyses were conducted in Stata 15.1. $P<0.05$ were considered as statistically significant.

\section{Results}

Table 1 describes the study sample characteristics by gender. The mean age of the participants was 39.4 years. The mean BMI for women $\left(29.5 \mathrm{~kg} / \mathrm{m}^{2} \pm 6.1\right)$ was higher than that for men $\left(28.4 \mathrm{~kg} / \mathrm{m}^{2} \pm 5.0\right)$. The prevalence of metabolic syndrome was $18.0 \%$ in both genders. Women had higher total fat mass, total fat percent, and trunk fat percent compared to men $(P<0.001)$. Visceral fat mass and percent were higher in men than women $(1.3 \pm 0.8$ $\mathrm{kg}$ vs $0.8 \pm 0.5 \mathrm{~kg}$ and $1.4 \pm 0.7 \%$ vs $1.0 \pm 0.6 \%$ respectively) $(P$-value $<0.001)$.

The cut-off points for body fat are presented in Table 2. Total fat mass of $34.0 \mathrm{~kg}$ was associated with cardiometabolic risk among men and women under the age of 40 with AUC of 0.7 and 0.8 for men and women respectively. For individuals aged $\geq 40$, total fat (AUC) of $30.6 \mathrm{~kg}(0.7)$ and $35.6 \mathrm{~kg}$ (0.6) was associated with 
Table 1 Sample characteristic by genders

\begin{tabular}{|c|c|c|c|c|}
\hline & \multirow{2}{*}{$\begin{array}{l}\text { Total } \\
\boldsymbol{N}=2407\end{array}$} & \multirow{2}{*}{$\begin{array}{l}\text { Male } \\
\boldsymbol{N}=1269\end{array}$} & \multirow{2}{*}{$\begin{array}{l}\text { Female } \\
\mathbf{N}=1138\end{array}$} & \multirow{2}{*}{$\begin{array}{l}\boldsymbol{P} \text { - } \\
\text { value }\end{array}$} \\
\hline & & & & \\
\hline Age (years) & $39.4(11.2)$ & $38.7(10.6)$ & $40.1(11.7)$ & 0.002 \\
\hline Education & & & & $<0.001$ \\
\hline Low & 239 (9.9\%) & $96(7.6 \%)$ & $143(12.6 \%)$ & \\
\hline Medium & $680(28.3 \%)$ & $388(30.6 \%)$ & $292(25.7 \%)$ & \\
\hline High & $1486(61.8 \%)$ & 783 (61.8\%) & $703(61.8 \%)$ & \\
\hline BMI (kg/m2) & $29.0(5.6)$ & $28.4(5.0)$ & $29.5(6.1)$ & $<0.001$ \\
\hline BMI categories & & & & $<0.001$ \\
\hline Normal & $575(23.9 \%)$ & 308 (24.3\%) & 267 (23.5\%) & \\
\hline Overweight & $900(37.4 \%)$ & 545 (42.9\%) & 355 (31.2\%) & \\
\hline Obese & $932(38.7 \%)$ & 416 (32.8\%) & $516(45.3 \%)$ & \\
\hline Metabolism syndrome & $434(18.0 \%)$ & 229 (18.0\%) & $205(18.0 \%)$ & 0.98 \\
\hline Total mass (kg) & $79.2(16.3)$ & $84.4(15.1)$ & $73.5(15.7)$ & $<0.001$ \\
\hline Total fat (kg) & $30.9(10.7)$ & $28.6(10.3)$ & $33.4(10.6)$ & $<0.001$ \\
\hline Total fat percent & $38.6(8.7)$ & $33.4(7.3)$ & $44.5(5.9)$ & $<0.001$ \\
\hline Trunk fat (kg) & $16.2(6.4)$ & $16.2(6.6)$ & $16.2(6.2)$ & 0.98 \\
\hline Trunk fat percent & $20.0(5.0)$ & $18.8(5.1)$ & $21.4(4.5)$ & $<0.001$ \\
\hline Visceral fat (kg) & $1.0(0.7)$ & $1.3(0.8)$ & $0.8(0.5)$ & $<0.001$ \\
\hline Visceral fat percent & $1.2(0.7)$ & $1.4(0.7)$ & $1.0(0.6)$ & $<0.001$ \\
\hline
\end{tabular}

Data are presented as mean and standard deviation (SD) for continuous measures, and $\mathrm{n}(\%)$ for categorical measures. Significance was set at $P<0.05$

cardiometabolic risk among men and women, respectively. The sensitivity of total fat, for those aged $<40$ was higher among women (82.8\%) compared to men (67.6\%). Specificity of total fat for those aged $<40$ was higher in men $(79.0 \%)$ compared to women (69.5\%). Men's sensitivity and specificity for total fat for those aged $\geq 40$ were higher in comparison to those for women in the same age range.
With regard to percentage body fat, the percentage body fat cut-off points (AUC) for men were $35.1 \%(0.7)$ and $34.8 \%$ (0.7) among those $<40$ and $\geq 40$ years, respectively. The corresponding figures for women were $45.1 \%(0.7)$ and $46.3 \%(0.6)$, respectively. Among men age $<40$, percentage fat sensitivity was lower $(71.7 \%)$ than for women $(79.3 \%)$ in the same age range. However, among women age $<40$, percentage fat specificity was less $(62.8 \%)$ than that for men $(66.2 \%)$ in same age range. The sensitivity and specificity of percentage fat for those aged $\geq 40$ in women (57.6 and 57.1\%) were much lower than those for men $(70.6$ and $60.1 \%)$ of the same age range, respectively.

The cut-off point (AUC) of trunk fat and trunk fat percent among men aged $<40$ were $20.9 \mathrm{~kg}(0.8)$ and $19.5 \%$ (0.7) respectively. Corresponding values (AUC) for men aged $\geq 40$ were $18.9 \mathrm{~kg}(0.7)$ and $21.6 \%(0.7)$ respectively. In women who were less than 40 years of age, the cut-off points (AUC) of truck fat (kg) and trunk fat $\%$ were $15.6 \mathrm{~kg}(0.8)$ and $22.4 \%$ (0.7). However, the corresponding values (AUC) for women with age of 40 and above were, $18.0 \mathrm{~kg}(0.7)$ and $23.4 \mathrm{~kg}(0.7)$.

The cut-off points (AUC) for visceral fat mass associated with metabolic risk among men and women with less than 40 years of age were $1.2 \mathrm{~kg}(0.8)$ and $0.8 \mathrm{~kg}(0.8)$ respectively. However, the corresponding values for men and women aged $\geq 40$ were $2.0 \mathrm{~kg}(0.8)$ and $1.0 \mathrm{~kg}(0.7)$ respectively. Considering the visceral fat percent, the cut-off values (AUC) were as follows 1.4\% (0.7) and 1.9\% (0.7) for men aged $<40$ years and men ages $\geq 40$ years respectively; and $1.0 \%(0.8)$ and $1.4 \%(0.7)$ for women aged $<40$ years and women ages $\geq 40$ years respectively.

\section{Discussion}

This study was conducted to develop gender and age specific cut-off points of BF\% for healthy Qatari adults

Table 2 Age-and-gender specific cutoff points for total fat associated with metabolic risk

\begin{tabular}{|c|c|c|c|c|c|c|c|c|}
\hline Variable & $\begin{array}{l}\text { Cut-point } \\
\text { Men }\end{array}$ & Sensitivity & Specificity & $A \cup C$ & $\begin{array}{l}\text { Cut-point } \\
\text { Women }\end{array}$ & Sensitivity & Specificity & $A \cup C$ \\
\hline Total fat, Age $<40$ & 34.0 & 67.6 & 79.0 & 0.7 & 34.0 & 82.8 & 69.5 & 0.8 \\
\hline Total fat, Age $\geq 40$ & 30.6 & 67.8 & 66.2 & 0.7 & 35.6 & 62.5 & 59.5 & 0.6 \\
\hline Percent fat, Age $<40$ & 35.1 & 71.6 & 66.2 & 0.7 & 45.1 & 79.3 & 62.8 & 0.7 \\
\hline Percent fat, Age $\geq 40$ & 34.8 & 70.6 & 60.1 & 0.7 & 46.3 & 57.6 & 57.1 & 0.6 \\
\hline Trunk fat, Age $<40$ & 20.8 & 67.6 & 84.7 & 0.8 & 15.7 & 86.2 & 68.3 & 0.8 \\
\hline Trunk fat, Age $\geq 40$ & 18.9 & 69.0 & 72.7 & 0.7 & 18.0 & 68.5 & 66.6 & 0.7 \\
\hline Trunk fat percentage, Age $<40$ & 19.5 & 75.7 & 69.4 & 0.7 & 22.4 & 69.0 & 77.0 & 0.7 \\
\hline Trunk fat percentage, Age $>=40$ & 21.6 & 66.7 & 72.7 & 0.7 & 23.4 & 65.8 & 66.1 & 0.7 \\
\hline Visceral fat, Age < 40 & 1.2 & 78.1 & 73.1 & 0.8 & 0.8 & 85.7 & 81.9 & 0.9 \\
\hline Visceral fat, Age $\geq 40$ & 2.0 & 64.8 & 83.8 & 0.7 & 1.0 & 75.7 & 67.0 & 0.7 \\
\hline Viseral fat percentage, Age $<40$ & 1.4 & 71.2 & 74.1 & 0.7 & 1.0 & 85.7 & 81.2 & 0.8 \\
\hline Viseral fat percentage, Age $>=40$ & 1.9 & 72.9 & 64.9 & 0.7 & 1.4 & 66.9 & 74.8 & 0.7 \\
\hline
\end{tabular}

Sensitivities and specificities are expressed in percentage (\%), and cut-off values are expressed in kg. AUC Area under the curve expressed in percentage (\%) 
to determine their risk of having metabolic syndrome. These cut-off points of BF\% have been created based on DEXA, anthropometric and biochemical data that have been collected from the study participants.

Body fat percentage is a better indicator of metabolic risk factors than body mass index [12]. The ability of BMI to diagnose obesity and risk of disease remains questionable [13]. The accuracy of BMI is limited due to the inability to measure body fat percentage directly from total body fat, total body lean mass, and total bone mass [14]. Different ethnic groups showed different results based on age and gender [15]. Therefore, additional cross-ethnic studies are required, as body fat percentage and BMI relations are affected by ethnicity, age and gender [16].

A study carried out on middle-aged Japanese men showed a link between BF\% and CVD risks, such as diabetes mellitus, dyslipidaemia and hypertension [17]. Li et al. (2012) revealed that there is a relationship between $\mathrm{BF} \%$, type 2 diabetes, and metabolic syndrome. They used BF\% from the Shanghai Diabetes Studies, which were $25 \%$ for men and $35 \%$ for women [14].

In our study, BF \% cut-off values that were associated with metabolic syndrome for Qatari adults aged $<40$ years old and $\geq 40$ years old were 35.1 and $45.1 \%$ for men, and 34.8 and $46.3 \%$ for women. The result obtained from BF\% showed a clearer link to metabolic syndrome risk for women, compared to men. This could be due to higher fat mass and lower lean body mass in women than men. Furthermore, women aged < 40 years had higher $\mathrm{BF} \%$ area under the curves (AUC) for CVD risk factors than women age $>40$ years. This high AUC of women $<40$ years old means that the results are diagnostic for a large number of women in this age range, thus giving higher predictability for metabolic risk and allowing early intervention to be initiated.

One of many similar studies is a study conducted by $\mathrm{Li}$ et al. in 2017 aimed at determining cut-off values of $\mathrm{BF} \%$ to estimate the risk of cardiovascular disease [14]. This was a cross-sectional study which included 3221 Chinese adults from two different ethnicities (2308 Han and 913 Mongolian). The study included 898 Han men and 355 Mongolian men, and 1410 Han women and 558 Mongolian women, with an age range of 20-80 years old. The BF\% cut-off points were measured using bioelectrical impedance. In the Mongolian population, men had body fat cut-off points of $25 \%$ and women had cutoff points of $35 \%$. The results were similar for the Han population [14]. Other studies in Korea supported the fact that $\mathrm{BF} \%$ is a strong indicator for cardio-metabolic risk [18]. Kim et al. stated that BF\% cut-off points in Korean populations are 21 and 37\% for men and women, respectively [18]. Moreover, another study has established individualised BF\% cut-off values for the AsianIndian people, reporting that men have a cut-off point of $24.5 \%$, while that of women is $38 \%$ [19].
The present study's results of trunk fat indicated that the specificity of trunk fat to predict metabolic risk was higher in males aged $<40(84.7 \%)$ than females aged < $40(68.2 \%)$; whereas the sensitivity was higher for women (86.2) than in men (67.6) at age $<40$ years.

One of the studies related to visceral fats is a study that involved 278 girls and 302 boys between the age of 3-19 years old [20]. Analysis of trunk fat was accomplished by DEXA. Specificity and sensitivity of trunk fat in boys were 92 and $87 \%$, and in girls were 94 and $89 \%$ [20]. Researchers have stated that the difference between boys and girls might be attributed to differences in body construction (apple shape, gynoid shape), in addition to the specific skeletal structure of each gender.

Snijder and co-workers (2004) conducted a study to investigate the association between trunk fat and blood glucose levels. They revealed that there is a strong correlation between trunk fat and metabolic syndromes, such as diabetes [21]. Meanwhile, Taylor et al., (2012) have reported that abdominal adiposity increases the risk of diabetes and cardiovascular disease based on their study of the Indian population [22]. Trunk fat in this population was measured using DEXA that measured fat in the abdominal area, such as the liver, subcutaneous fat and other tissues as well [22].

Our results revealed the visceral fat cut-off points associated with metabolic risks were slightly higher in men in comparison to those of women in both age ranges, with $1.2 \mathrm{~kg}$ visceral fat cut-off value in men and $0.8 \mathrm{~kg}$ cut-off value for women under the age of 40. A result, $2.0 \mathrm{~kg}$ of visceral fat has been indicated as the borderline for men $\geq 40$ to get a metabolic disease, while the borderline for women $\geq 40$ is $1.0 \mathrm{~kg}$ of visceral fat.

One of the studies related to visceral fat is a crosssectional study conducted by Amato et al., (2011) among Caucasian Sicilian subjects. The sample size was 1764 primary care (PC) patients, of whom 585 were men and 1179 were women, aged between 16 and 99 years old [23]. The researchers have indicated a strong relationship between the visceral adiposity index (VAI) and metabolic risk factors. In primary care patients $<30$ years, $30-42$ years, $42-$ 52 years, $52-66$ years, $>66$ years, VAI cut off points were $2.52,2.23,1.92,1.93$, and 2 , respectively [23].

Trunk and visceral fat percentages - unlike the trunk and visceral fat mass- may provide information about the visceral fat and trunk fat in relation to the total body mass. However, our analysis did not show a privilege of using percentage of fat over using mass of fat (specificity and sensitivity were comparable).

\section{Conclusion}

This study established specific cut-off values of body fat parameters for Qatari adults of different ages and gender that can be used as a reference for determining obesity- 
related metabolic risks. The study has revealed that there is a strong relation between body fatness and the risk of metabolic diseases.

\section{Acknowledgements}

Acknowledgement is due to Qatar Biobank for providing the data with all required support. This research is funded by Qatar University.

\section{Authors' contributions}

HB: conceptualization of the research, supervising students, writing manuscript. SH: results interpretation, drafting the manuscript. AZ: results interpretation, drafting the manuscript. HS: results interpretation, drafting the manuscript. AK: drafting the manuscript. JAT: drafting the manuscript. ZS: statistical analysis; drafting the manuscript. The authors read and approved the final manuscript

\section{Funding}

This research is funded by Qatar University. J.A. Tur is funded by CIBEROBN (CB12/03/30038), Instituto de salud Carlos III, Spain and European Regional Development Fund

\section{Availability of data and materials}

The research is based on secondary data owned by Qatar Biobank. Authors do not have the authority to provide the data.

\section{Ethics approval and consent to participate}

This study was conducted according to the guidelines laid down in the Declaration of Helsinki and all procedures involving research study participants were approved by the Qatar Biobank ethics committee. Written informed consent was obtained from all subjects.

\section{Consent for publication}

N/A

\section{Competing interests}

Authors declare No conflict of interest.

\section{Author details}

'Department of Human Nutrition, College of Health Sciences, QU Health, Qatar University, Doha 2713, Qatar. ${ }^{2}$ Research Group on Community Nutrition \& Oxidative Stress, University of the Balearic Islands \& CIBEROBN 07122 Palma de Mallorca, Spain.

Received: 30 December 2019 Accepted: 20 May 2020

Published online: 25 July 2020

\section{References}

1. Bastien M, Poirier P, Lemieux I, Després J-P. Overview of epidemiology and contribution of obesity to cardiovascular disease. Prog Cardiovasc Dis. 2014; 56:369-81

2. Cheema S, Mamtani R, Lowenfels A, Alrouh H, Al-Samraye S, Ismail A, Abdulmalik M, Aigha II, Aseel MT, El Emadi N. Obesity in the Qatari population: an epidemiological perspective. In: Qatar Foundation annual research conference proceedings volume 2016 issue 1: Hamad bin Khalifa University Press (HBKU Press); 2016. HBPP2046.

3. Gallagher EJ, LeRoith D. Obesity and diabetes: the increased risk of cancer and cancer-related mortality. Physiol Rev. 2015;95:727-48.

4. Petrie JR, Guzik TJ, Touyz RM. Diabetes, hypertension, and cardiovascular disease: clinical insights and vascular mechanisms. Can J Cardiol. 2018;34: 575-84.

5. Re RN. Obesity-related hypertension. Ochsner J. 2009;9:133-6.

6. Carpenter $\mathrm{CL}$, Yan E, Chen S, Hong K, Arechiga A, Kim WS, et al. Body fat and body-mass index among a multiethnic sample of college-age men and women. J Obes. 2013;2013:1.

7. Al Dokhi L, Shahid Habib S. Assessment of gender differences in body composition and physical fitness scoring in Saudi adults by bioelectrical impedance analysis. Acta Clin Croatica. 2013:52:189-94.

8. Wang D, Li Y, Lee SG, Wang L, Fan J, Zhang G, et al. Ethnic differences in body composition and obesity related risk factors: study in Chinese and white males living in China. PLoS One. 2011;6:e19835.
9. Al Kuwari $\mathrm{H}, \mathrm{Al}$ Thani A, Al Marri A, Al Kaabi A, Abderrahim $\mathrm{H}$, Afifi N, et al. The Qatar biobank: background and methods. BMC Public Health. 2015;15: 1208

10. Alberti $\mathrm{K}$, Eckel RH, Grundy SM, Zimmet PZ, Cleeman Jl, Donato KA, et al. Harmonizing the metabolic syndrome: a joint interim statement of the international diabetes federation task force on epidemiology and prevention; national heart, lung, and blood institute; American heart association; world heart federation; international atherosclerosis society; and international association for the study of obesity. Circulation. 2009;120:16405.

11. Liu X. Classification accuracy and cut point selection. Stat Med. 2012;31: 2676-86.

12. Ramírez-Vélez R, Correa-Bautista J, Sanders-Tordecilla A, Ojeda-Pardo M, Cobo-Mejía E, Castellanos-Vega R, et al. Percentage of body fat and fat mass index as a screening tool for metabolic syndrome prediction in Colombian university students. Nutrients. 2017;9:1009.

13. Deurenberg P. Universal cut-off BMI points for obesity are not appropriate. Br J Nutr. 2001:85:135-6.

14. Li Y, Wang H, Wang K, Wang W, Dong F, Qian Y, et al. Optimal body fat percentage cut-off values for identifying cardiovascular risk factors in Mongolian and Han adults: a population-based cross-sectional study in Inner Mongolia, China. BMJ Open. 2017;7:e014675.

15. Deurenberg P, Yap M, Van Staveren WA. Body mass index and percent body fat: a meta analysis among different ethnic groups. Int J Obes. 1998; 22:1164.

16. Deurenberg P, Deurenberg-Yap M. Validity of body composition methods across ethnic population groups. Acta Diabetol. 2003;40:s246-9.

17. Yamashita K, Kondo T, Osugi S, Shimokata K, Maeda K, Okumura N, et al. The significance of measuring body fat percentage determined by bioelectrical impedance analysis for detecting subjects with cardiovascular disease risk factors. Circ J. 2012:76:2435-42.

18. Kim C-H, Park HS, Park M, Kim H, Kim C. Optimal cutoffs of percentage body fat for predicting obesity-related cardiovascular disease risk factors in Korean adults. Am J Clin Nutr. 2011;94:34-9.

19. Joseph L, Wasir JS, Misra A, Vikram NK, Goel K, Pandey RM, et al. Appropriate values of adiposity and lean body mass indices to detect cardiovascular risk factors in Asian Indians. Diabetes Technol Ther. 2011:13: 899-906.

20. Taylor RW, Jones IE, Williams SM, Goulding A. Evaluation of waist circumference, waist-to-hip ratio, and the conicity index as screening tools for high trunk fat mass, as measured by dual-energy X-ray absorptiometry, in children aged 3-19 y. Am J Clin Nutr. 2000;72:490-5.

21. Snijder MB, Dekker JM, Visser M, Bouter LM, Stehouwer CD, Yudkin JS, et al. Trunk fat and leg fat have independent and opposite associations with fasting and postload glucose levels: the Hoorn study. Diabetes Care. 2004; 27:372-7.

22. Taylor AE, Kuper H, Varma RD, Wells JC, Bell JD, Radhakrishna K, et al. Validation of dual energy X-ray absorptiometry measures of abdominal fat by comparison with magnetic resonance imaging in an Indian population. PLoS One. 2012;7:e51042

23. Amato MC, Giordano C, Pitrone M, Galluzzo A. Cut-off points of the visceral adiposity index (VAI) identifying a visceral adipose dysfunction associated with cardiometabolic risk in a Caucasian Sicilian population. Lipids Health Dis. 2011;10:183.

\section{Publisher's Note}

Springer Nature remains neutral with regard to jurisdictional claims in published maps and institutional affiliations. 